\title{
Cerebral haemodynamics and depression in the elderly
}

\author{
H Tiemeier, S L M Bakker, A Hofman, P J Koudstaal, M M B Breteler
}

J Neurol Neurosurg Psychiatry 2002;73:34-39

See end of article for authors' affiliations

.....................

Correspondence to: $\operatorname{Dr} M M B$ Breteler,

Department of

Epidemiology \&

Biostatistics, Erasmus

Medical Centre Rotterdam,

PO Box 1738, 3000 DR

Rotterdam, Netherlands;

breteler@epib.fgg.eur.nl

Received

3 September 2001

In revised form

13 March 2002

Accepted 13 March 2002

\begin{abstract}
Background: Evidence from epidemiological and neuroimaging studies suggests that cerebrovascular disease is associated with depressive disorders in the elderly, but the extent to which it contributes to the pathogenesis of late life depression is unclear.

Objective: To investigate the relation between cerebral haemodynamics and depression in a population based study, using transcranial Doppler ultrasonography.

Methods: Cerebral blood flow velocity and $\mathrm{CO}_{2}$ induced vasomotor reactivity in the middle cerebral artery were measured in 2093 men and women who participated in the Rotterdam study. All subjects were screened for depressive symptoms using the Center of Epidemiological Studies Depression scale, and those with a score of 16 or over had a psychiatric work up. In a semistructured interview, diagnoses of depressive disorders according to the DSM-IV and subthreshold depressive disorder were established. Analyses of covariance controlled for age, sex, stroke, cognitive score, and cardiovascular risk factors were used to compare means of haemodynamic variables.

Results: Subjects with depressive symptoms had reduced blood flow velocities (mean difference, -2.9 $\mathrm{cm} / \mathrm{s} ; 95 \%$ confidence interval $(\mathrm{Cl}),-5.0$ to $-0.8 ; \mathrm{p}=0.008$ ) and lower vasomotor reactivity (mean difference $-0.5 \% / \mathrm{kPa} ; 95 \% \mathrm{Cl},-1.0$ to $-0.05 ; \mathrm{p}=0.03)$. Blood flow velocity was reduced most in subjects suffering from a DSM-IV depressive disorder (mean difference, $-4.9 \mathrm{~cm} / \mathrm{s} ; 95 \% \mathrm{Cl},-8.5$ to $-1.4 ; p=0.006)$. The overall reduction in vasomotor reactivity was accounted for by subjects with subthreshold depressive disorder.

Conclusions: Depression in late life is associated with cerebral haemodynamic changes that can be assessed by transcranial Doppler ultrasonography. The observed reduction in cerebral blood flow velocity could be a result of reduced demand in more seriously depressed cases with a DSM-IV disorder, whereas reduced $\mathrm{CO}_{2}$ induced cerebral vasomotor reactivity is a possible causal factor for subthreshold depressive disorder.
\end{abstract}

$\mathrm{T}$ he "vascular depression" hypothesis postulates that in late life vascular pathology contributes significantly to the pathogenesis of depression. ${ }^{1}$ Evidence from epidemiology, genetics, and neuropsychology suggests that cerebrovascular changes may lead to depressive disorders. ${ }^{2-6}$ Strong evidence comes in particular from neuroimaging studies. ${ }^{27}$ These studies have focused on magnetic resonance imaging (MRI) hyperintensities, but single photon emission tomography (SPECT) and positron emission tomography (PET) have also been used to provide information on the function of the brain in depressive disorders. ${ }^{8-10}$ In studies using MRI, white matter hyperintensities in the basal ganglia and the frontal lobes were reported. These lesions are attributed to vascular disease, but other causes cannot be ruled out. ${ }^{1}$ In clinical studies employing SPECT, regional differences in cerebral blood flow were reported between subjects with depressive disorders and clinical controls. ${ }^{1-14}$ It remains unclear whether low blood flow is a result of depression, reflects cerebrovascular disease, or marks neuronal loss predisposing to depression. ${ }^{15}$

With transcranial Doppler ultrasonography it is possible to assess the intracranial circulation directly. Aaslid et al first described a non-invasive Doppler ultrasound technique in 1982, which took advantage of the relatively thin temporal bone. ${ }^{16}$ Transcranial Doppler is a useful tool for detecting the haemodynamic changes resulting from cerebrovascular impairment, despite the limitation that the temporal bone often cannot be passed by ultrasound. ${ }^{17}{ }^{18}$ Changes in both vasomotor reactivity and blood flow velocity are commonly measured in the basal cerebral arteries by transcranial Doppler. Vasomotor reactivity is reduced in patients with cerebral microangiopathy, ${ }^{17}$ while changes in cerebral artery blood velocities reflect changes in blood flow and brain tissue perfusion. ${ }^{19-21}$
The Rotterdam study provided an opportunity to examine the relation between cerebral haemodynamics and depression in a population based sample of older adults. We conducted this study to evaluate whether cerebrovascular impairment, as measured by transcranial Doppler, is related to depression. Our a priori hypothesis was that subjects with depressive disorders have a reduced flow velocity and reduced vasomotor reactivity.

\section{METHODS}

\section{Subjects}

The investigation was conducted as part of the Rotterdam study, a population based cohort study ongoing since 1990, in which all inhabitants aged 55 and over in a suburb of Rotterdam were invited to participate. ${ }^{22}$ In the third survey we added assessments of depressive symptoms and cerebral haemodynamics to the protocol. Measurements were conducted between 1997 and 1999. Screening for depressive symptoms was carried out during the home interview part of the survey, in which 4730 subjects participated.

In 3101 consecutive participants we attempted to perform transcranial Doppler ultrasonography as part of the standard clinical investigation at the research centre. No transcranial Doppler measurements were carried out in the remainder ( $\mathrm{n}=1629$ ), owing to the unavailability of a technician. In 990 subjects $(32 \%)$, transcranial Doppler was undertaken but no results were obtained. In most cases this was because of window failure on both sides $(n=771)$ or because restlessness or discomfort prevented the study being done $(n=36)$. In 183 participants haemodynamic measurements could not be carried out for other reasons, such as ambiguous flow direction or lack of time. ${ }^{19}{ }^{23}$ Subjects in whom no transcranial 
Doppler data were obtained were older $(\mathrm{p}<0.001)$ and more likely to be female $(p<0.001)$. The prevalence of psychiatric symptoms in the analytical sample was found to be lower than in the study population as a whole $(5.5 \% v 7.8 \%)$. A further 18 subjects were excluded because they did not have complete screening for depression. The final study sample consisted of 2093 participants in whom adequate depression and haemodynamic indices were obtained.

The Rotterdam Study was approved by the medical ethics committee of Erasmus University Medical School. After complete description of the study to the subjects, written informed consent was obtained.

\section{Depression assessment}

Depressive disorders were assessed using a two step procedure. First, participants completed the Dutch version of the original Center for Epidemiology Studies Depression scale (CES-D) during the home interview. The CES-D is a 20 item self reported measure of symptoms experienced in the past week, scored on a scale of 0 to 3 points. The validity of the CES-D has been well established. ${ }^{24}$ We used a score of $\geqslant 16$ as a cut off point, as this has been found to have a high sensitivity for major depression in a random sample of older subjects in the Netherlands. ${ }^{25}$ Previous studies have verified that a score of 16 and above on the CES-D represents clinically significant depressive symptoms. ${ }^{26}$

In a second step, screen positive subjects had a psychiatric work up. They were evaluated by the Dutch version of the present state examination (PSE-10), a semistructured psychiatric interview included in the Schedules for Clinical Assessment in Neuropsychiatry. ${ }^{27}$ All interviews were conducted by two experienced clinicians. Psychiatric disorders were classified according to the DSM-IV criteria, with an algorithm based on the PSE-10 scores. The diagnostic categories include minor depression, as defined in the appendix of DSM-IV.

Of the 2093 subjects included in the analyses, 116 (5.5\%) were screen positive for depression as measured by the CES-D. A psychiatric work up was performed in 111 of these participants $(95.6 \%)$. Four subjects refused to participate in this evaluation, and one screen positive subject could not be reached. A depressive disorder as defined by the DSM-IV criteria was established in 42 cases. The remaining subjects were either classified as having anxiety disorders or other psychiatric disease $(n=8)$ or did not meet criteria for an axis I psychiatric disorder $(n=61$, subthreshold depressive disorder).

To define late onset depression we used the data from the baseline interview with a physician. All subjects in the present analysis responded to the questions about psychiatric history. Participants who reported a history of depression before the age of 60 were considered to be suffering from early onset depression.

\section{Transcranial Doppler assessment}

Transcranial Doppler ultrasonography was done using a Multi-Dop X-4 instrument (DWL, Sipplingen, Germany), and the cerebral blood flow velocity $(\mathrm{cm} / \mathrm{s})$ was measured in the middle cerebral artery on both sides if possible. End diastolic, peak systolic, and mean cerebral blood flow velocity were recorded automatically.

$\mathrm{CO}_{2}$-induced cerebral vasomotor reactivity measurements were done as follows. The cerebral blood flow velocity was measured continuously and the participants first breathed room air through an anaesthetic mask, tightly fitted over mouth and nose, until a steady expiratory end tidal $\mathrm{CO}_{2}$ was obtained. Next, participants inhaled a mixture of 5\% carbon dioxide in 95\% oxygen for two minutes. Cerebral vasomotor reactivity was defined as the percentage increase in cerebral blood flow velocity occurring during inspiration of $5 \% \mathrm{CO}_{2}$, divided by the absolute increase in end tidal $\mathrm{CO}_{2}$ in the same period $(\% \mathrm{kPa})$. End tidal $\mathrm{pCO}_{2}(\mathrm{kPa})$ was recorded continuously with a $\mathrm{CO}_{2}$ analyser (Multinex, Datascope, Hoevelaken, Netherlands). End expiratory $\mathrm{CO}_{2}$ was assumed to reflect arterial $\mathrm{CO}_{2}$. TCD-8 DWL special software $\left(\mathrm{VMR}-\mathrm{CO}_{2}\right)$ was used. All transcranial Doppler data were stored on hard disc for off-line analysis. The mean of the right and left haemodynamic variables was used for analyses if both middle cerebral arteries could be insonated adequately. A one sided haemodynamic variable was used if there was unilateral window failure.

\section{Measurements of other covariates}

The following were considered to be possible confounding variables: age, sex, education, cognitive function (measured by the mini mental state examination (MMSE)), antihypertensive treatment, and antidepressant treatment. Education was measured on an ordinal scale and later dichotomised into low and high. Information on current antidepressant or antihypertensive drug treatment was obtained during the home interview. The following cardiovascular risk factors were assessed: stroke, smoking, systolic and diastolic blood pressure, diabetes mellitus, and total cholesterol. A history of stroke was obtained from all subjects through direct questioning and computerised linkage with general practitioner medical records. The history was considered positive when confirmed by a physician. Cigarette smoking was analysed in categories of current and former smoker. Sitting blood pressure was measured twice on the right arm with a random zero sphygmomanometer. Diabetes mellitus was defined as the use of insulin or oral blood glucose lowering drugs, or serum glucose concentrations of more than $11.0 \mathrm{mmol} / \mathrm{l}$. Fasting blood samples were taken and serum total cholesterol was measured using an automated enzymatic procedure. The ankle to brachial index was used as an indicator of peripheral atherosclerosis. We assessed ankle to brachial index by taking the ratio of the systolic blood pressure measured at the tibial artery to that measured at the right arm. Subjects with an ankle to brachial index of less than 0.9 were considered to suffer from peripheral arterial disease. ${ }^{28}$

\section{Statistical analysis}

The associations between haemodynamic variables and depressive disorders were addressed in three ways.

First, analysis of covariance (ANCOVA) was used to calculate means of the screen positive subjects and the reference group, adjusted for age and sex. Haemodynamic indices were entered as continuous variables. In addition, we controlled these analyses for education, antihypertensive and antidepressant drug treatment, cognitive function, and cardiovascular risk factors. We also performed an ANCOVA to evaluate possible differences between subjects with depressive disorders and subjects with subclinical depressive symptoms. Subjects without a psychiatric work up and those with other psychiatric disorders were excluded from subgroup analyses. Analyses were run both excluding and adjusting for subjects with a history of stroke ( $\mathrm{n}=7$ in the screen positive group, including two subjects with depressive disorder) or taking antidepressant drugs.

Second, logistic regression analysis was used to calculate the odds ratios for the association between haemodynamic variables and the presence of depressive disorders. We assessed tertiles of haemodynamic variables to allow for a non-linear relation. Because of the relatively small number of cases we did not use more categories. In this model we included only the variables associated with haemodynamic indices to avoid an overfitted model.

Third, we used stratified analyses to study possible effect modification by peripheral arterial disease and a history of depression. 
Table 1 Characteristics of participants with and without depressive symptoms

\begin{tabular}{|c|c|c|}
\hline & Non-depressed $(n=1977)$ & CES-D score $\geqslant 16(n=116)$ \\
\hline Age (years) & $71.1(6.5)$ & $72.8(6.6)^{* *}$ \\
\hline Male & $54.1 \%$ & $41.4 \% * *$ \\
\hline Primary education only & $42 \%$ & $58 \% * *$ \\
\hline Previous stroke & $2.3 \%$ & $6.0 \% *$ \\
\hline Diabetes & $6 \%$ & $5 \%$ \\
\hline \multicolumn{3}{|l|}{ Smoking } \\
\hline Current smoker & $16 \%$ & $21 \%$ \\
\hline Former smoker & $56 \%$ & $47 \%$ \\
\hline MMSE score & $27.9(1.8)$ & $27.1(2.6)^{* *}$ \\
\hline Systolic blood pressure $(\mathrm{mm} \mathrm{Hg})$ & $143(18)$ & $142(20)$ \\
\hline Diastolic blood pressure $(\mathrm{mm} \mathrm{Hg})$ & $75(11)$ & $74(12)$ \\
\hline Total cholesterol (mmol/l) & $5.8(1.0)$ & $5.8(1.0)$ \\
\hline Antidepressant drug treatment & $1.7 \%$ & $10.5 \%$ ** \\
\hline Antihypertensive drug treatment & $35 \%$ & $33 \%$ \\
\hline Peripheral arterial disease & $17 \%$ & $25 \%$ \\
\hline Major depression before age 60 & $3.5 \%$ & $6.9 \% *$ \\
\hline \multicolumn{3}{|c|}{$\begin{array}{l}\text { Values are unadjusted mean (SD) or percentages } \\
{ }^{*} p<0.05 ;{ }^{* *} p<0.001 \text {, adjusted for age and sex were appropriate. } \\
\text { MMSE, mini mental state examination. }\end{array}$} \\
\hline
\end{tabular}

\section{RESULTS}

Table l compares the demographic characteristics and confounding variables of the 116 participants who were screen positive and the 1977 participants who were screen negative for depression. Age, sex, education, a history of major depression before age 60 , previous stroke, and cognitive function were all associated with current depressive symptoms. However, the cardiovascular risk factors smoking, diabetes, systolic and diastolic blood pressure, and total cholesterol were not related to depressive symptoms.

Subjects with depressive symptoms as determined by the CES-D had a lower mean cerebral blood flow velocity (age and sex adjusted mean difference, $-2.9 \mathrm{~cm} / \mathrm{s} ; 95 \%$ confidence interval (CI), -5.0 to $-0.8 ; \mathrm{p}=0.007$ ) and reduced vasomotor reactivity (age and sex adjusted mean difference, $-0.7 \% / \mathrm{kPa}$;
$95 \% \mathrm{CI},-1.2$ to $-0.2 ; \mathrm{p}=0.008$ ). Table 2 shows the relation between cerebral haemodynamic variables and depressive symptoms, with additional adjustment for education, history of stroke, antidepressant and antihypertensive drug treatment, cognitive function, and cardiovascular risk factors.

In a further analysis, screen positive subjects were classified according to the severity of the depressive symptoms (table 3). Subjects with subthreshold depressive disorder and DSM-IV depressive disorders were included as distinct groups. The results showed a consistent pattern for end diastolic, mean, and peak systolic blood flow velocity. Blood flow velocity of subjects with depressive disorders was significantly lower than in the reference group. The mean values of subjects with a subthreshold depressive disorder lay in between. A different pattern was observed for vasomotor reactivity. Subjects with

Table 2 Association between cerebral haemodynamic variables and depressive symptoms

\begin{tabular}{|c|c|c|c|c|}
\hline & \multirow{2}{*}{$\begin{array}{l}\text { Non-depressed }(n=1977) \\
\text { Mean }\end{array}$} & \multicolumn{3}{|c|}{ CES-D score $\geqslant 16(n=116)$} \\
\hline & & Mean & Adjusted difference $(95 \% \mathrm{Cl})$ & $\mathrm{p}$ Value \\
\hline \multicolumn{5}{|l|}{ Blood flow velocity $(\mathrm{cm} / \mathrm{s})$} \\
\hline End diastolic & 32.5 & 29.6 & $-2.3(-3.9$ to -0.7$)$ & 0.005 \\
\hline Mean & 50.5 & 47.8 & $-2.9(-5.0$ to -0.8$)$ & 0.008 \\
\hline Peak systolic & 86.5 & 82.5 & $-4.2(-7.6$ to -0.7$)$ & 0.02 \\
\hline Vasomotor reactivity $(\% / \mathrm{kPa})$ & 3.9 & 3.1 & $-0.5(-1.0$ to -0.05$)$ & 0.03 \\
\hline
\end{tabular}

Analysis of covariance with haemodynamic data entered as continuous variables and adjusted for age, sex, education, cognitive function, smoking, systolic and diastolic blood pressure, antidepressant and antihypertensive drug treatment, total cholesterol, diabetes mellitus, and history of stroke. Values are unadjusted means and adjusted differences, using pairwise comparison with non-depressed reference group.

$\mathrm{Cl}$, confidence interval; CES-D, Center for Epidemiology Studies depression scale.

Table 3 Association between cerebral haemodynamic variables and depression

\begin{tabular}{|c|c|c|c|c|c|c|c|}
\hline & \multirow{2}{*}{$\begin{array}{l}\begin{array}{l}\text { Non-depressed } \\
(n=1929)\end{array} \\
\text { Mean }\end{array}$} & \multicolumn{3}{|c|}{ Subthreshold depressive disorder $(n=59)$} & \multicolumn{3}{|c|}{ Depressive disorders $(n=40)$} \\
\hline & & Mean & Adjusted difference & $\mathrm{p}$ Value & Mean & Adjusted difference & $\mathrm{p}$ Value \\
\hline \multicolumn{8}{|l|}{ Blood flow velocity $(\mathrm{cm} / \mathrm{s})$} \\
\hline End diastolic & 32.5 & 30.6 & $-1.0(-3.1$ to 1.2$)$ & 0.37 & 27.8 & $-4.0(-6.6$ to -1.4$)$ & 0.003 \\
\hline Mean & 50.5 & 48.5 & $-2.0(-4.8$ to 0.9$)$ & 0.20 & 44.9 & $-4.9(-8.5$ to -1.4$)$ & 0.006 \\
\hline Peak systolic & 86.5 & 84.1 & $-4.0(-8.7$ to 0.8$)$ & 0.10 & 79.5 & $-6.8(-12.5$ to -1.0$)$ & 0.02 \\
\hline Vasomotor reactivity $(\% / \mathrm{kPa})$ & 3.9 & 2.7 & $-0.9(-1.6$ to -0.2$)$ & 0.007 & 3.4 & $-0.3(-1.1$ to 0.6$)$ & 0.52 \\
\hline
\end{tabular}

Analysis of covariance with haemodynamic data entered as continuous variables and adjusted for age, sex, education, cognitive function, smoking, systolic and diastolic blood pressure, antidepressant and antihypertensive drug treatment, total cholesterol, and diabetes mellitus. Subjects with stroke were excluded.

Values represent unadjusted means and adjusted differences ( $95 \%$ confidence interval), using pairwise comparison with a non-depressed reference group, same covariates. 
Table 4 The relation between tertiles of cerebral haemodynamic variables and depression expressed as odds ratios

\begin{tabular}{|c|c|c|c|c|}
\hline \multirow[b]{2}{*}{ Tertile } & \multirow[b]{2}{*}{ Number of subjects } & \multicolumn{3}{|c|}{ Odds ratios* $(95 \% \mathrm{Cl})$} \\
\hline & & $\begin{array}{l}\text { CES-D score } \geqslant 16 \\
(n=116)\end{array}$ & $\begin{array}{l}\text { Subthreshold depressive } \\
\text { disordert }(n=59)\end{array}$ & Depression $\ddagger(n=40)$ \\
\hline \multicolumn{5}{|c|}{ Mean blood flow velocity (range in $\mathrm{cm} / \mathrm{s}$ ) } \\
\hline 1 st $(54.4$ to 112$)$ & 692 & 1.0 (reference) & 1.0 (reference) & 1.0 (reference) \\
\hline 2nd (44.8 to 54.3$)$ & 697 & $1.2(0.7$ to 2.0$)$ & $1.1(0.6$ to 2.1$)$ & $1.2(0.5$ to 2.9$)$ \\
\hline 3 rd (14.8 to 44.7$)$ & 703 & $1.9(1.2$ to 3.1$)$ & $1.5(0.8$ to 2.7$)$ & $2.6(1.2$ to 5.8$)$ \\
\hline \multicolumn{5}{|c|}{ End diastolic blood flow velocity (range in $\mathrm{cm} / \mathrm{s}$ ) } \\
\hline 1 st $(35.6$ to 83.5$)$ & 694 & 1.0 (reference) & 1.0 (reference) & 1.0 (reference) \\
\hline 2 nd $(28.1$ to 35.5$)$ & 692 & $1.0(0.6$ to 1.7$)$ & $0.8(0.4$ to 1.6$)$ & $1.2(0.5$ to 2.8$)$ \\
\hline 3 rd $(8.0$ to 28.0$)$ & 705 & $1.8(1.1$ to 2.9$)$ & $1.2(0.7$ to 2.3$)$ & $2.5(1.1$ to 5.5$)$ \\
\hline \multicolumn{5}{|c|}{ Peak systolic blood flow velocity (range in $\mathrm{cm} / \mathrm{s}$ ) } \\
\hline $1 \mathrm{st}(93.6$ to 170$)$ & 695 & 1.0 (reference) & 1.0 (reference) & 1.0 (reference) \\
\hline 2nd (77.0 to 93.5$)$ & 692 & $1.3(0.8$ to 2.1$)$ & $1.2(0.6$ to 2.2$)$ & $1.2(0.5$ to 2.7$)$ \\
\hline 3rd (37.0 to 77.0$)$ & 706 & $1.7(1.0$ to 2.7$)$ & $1.5(0.8$ to 2.7$)$ & $2.0(0.9$ to 4.3$)$ \\
\hline \multicolumn{5}{|c|}{ Vasomotor reactivity (range in $\% / \mathrm{kPa}$ ) } \\
\hline lst $(4.48$ to 22.9$)$ & 669 & 1.0 (reference) & 1.0 (reference) & 1.0 (reference) \\
\hline 2 nd ( 2.67 to 4.47$)$ & 674 & $2.2(1.3$ to 3.8$)$ & $2.8(1.3$ to 6.4$)$ & $1.6(0.7$ to 3.5$)$ \\
\hline 3 rd $(0.05$ to 2.66$)$ & 671 & $2.0(1.2$ to 3.5$)$ & $3.0(1.3$ to 6.7$)$ & $1.2(0.5$ to 2.7$)$ \\
\hline
\end{tabular}

Note: the numbers of cases with subthreshold depressive disorder and depressive disorder do not add up to 116 because five subjects had no psychiatric work-up and 12 screen positive subjects had other psychiatric diseases or a previous stroke.

*Logistic regression adjusted for age, sex, and cognitive function.

†Subjects with depression or a history of stroke excluded from analysis.

$\ddagger$ Subjects with subthreshold depressive disorder or a history of stroke excluded from analysis.

subthreshold depressive disorder had a lower vasomotor reactivity than non-depressed reference subjects, whereas there was no clear cut difference between subjects with depressive disorders and the reference group. Very similar estimates were observed if we excluded subjects with a history of stroke or those taking antidepressant drugs, rather than adjusting for those variables.

Table 4 shows the odds ratios for depressive symptoms per tertile of haemodynamic variables adjusted for age, sex, and cognitive function. The particular contributions of subjects with subthreshold depressive disorder and subjects with DSM-IV depressive disorders are also presented in the table. It can be seen that more subjects with depressive disorders were found in the lowest tertile of blood flow velocity. For vasomotor reactivity, the middle and the lower tertiles were associated with an increased risk of subthreshold depressive disorder.

The observed relations between depressive status and cerebral haemodynamic variables were not altered after we controlled for a history of major depression before age 60 and peripheral arterial disease. There were more subjects with peripheral arterial disease in the group with subthreshold depressive disorder $(28.8 \%)$ and the group with depressive disorders $(25.0 \%)$ than in the reference group (16.5\%). However, the relation between blood flow velocity or vasomotor reactivity and depression was neither explained by nor modified by peripheral arterial disease (data not shown).

\section{DISCUSSION}

This study shows that haemodynamic changes as assessed by transcranial Doppler ultrasonography are associated with depressive symptoms. Both cerebral blood flow velocity and vasomotor reactivity were found to be lower in subjects with depressive symptoms, after the effects of age, sex, education, history of stroke, cognitive function, and cardiovascular risk factors were controlled for. These results support the view that cerebrovascular impairment may be a cause of depressive symptoms in the elderly. To our knowledge this study is the first to report results of transcranial Doppler ultrasound measurements and depression. Most studies using transcranial Doppler ultrasonography in psychiatric settings have looked at dementia or panic disorders. ${ }^{20} 29-33$

The strength of the present large population based study is the psychiatric work up in subjects who were screen positive on the CES-D. A previous study in an elderly Dutch population reported high sensitivity using the same cut off point, and misclassification of disease is thus unlikely to have influenced our results. ${ }^{25}$ Furthermore, we were able to determine in which group depressive symptoms were caused by depressive disorders. The prevalence of depressive symptoms in the Rotterdam study $(7.8 \%)$ falls within the range reported in a recent review of community prevalence of depressive symptoms in the elderly and is comparable with the prevalence of $9.0 \%$ reported in the USA. ${ }^{26}$

The term "vascular depression" was introduced by Alexopoulos. ${ }^{1}$ He postulated that geriatric depression encompasses a high percentage of patients with cerebrovascular disease. While there are few clinical differences between early and late onset depression, ${ }^{7}$ the hypothesis has been supported by studies showing that persons with late onset depression have more neuroradiological abnormalities than nondepressed individuals. ${ }^{34}$ This finding could only partially be replicated in a population based study. Steffens et al reported hyperintensities in the basal ganglia, but other white matter lesions were not related to depressive symptoms as measured by a shortened version of the CES-D. ${ }^{2}$

Different mechanisms for altered cerebral blood flow velocity as measured by transcranial Doppler have been postulated.$^{35}$ Reduced blood flow may reflect altered cerebral metabolism, an intrinsic property of the vascular smooth muscle, or a neuronal dysfunction of sympathetic nerve fibres. Metabolic autoregulation is probably of key importance and explains the increased flow velocity during cognitive activity. ${ }^{36}$ As reduced cognitive activity is a well recognised symptom of depressive disorder, a reduction of blood flow velocity might be an epiphenomenon of depression. The decreased blood flow velocity in our study could reflect the diminished demand in depressive states and does not necessarily support the vascular hypothesis.

Vasomotor reactivity, on the other hand, is probably a good indicator of microangiopathy. A reduced vasomotor reactivity indicates that the cerebral arterioles are unable to dilate in order to compensate for increased demand. ${ }^{38}$ In patients with stroke or transient ischaemic attacks reduced vasomotor reactivity has often been reported. ${ }^{17}{ }^{39}$ In a subset of 73 patients with MRI scans who participated in this study, we previously observed that vasomotor reactivity was related to deep subcortical and periventricular white matter lesions. ${ }^{40}$ However, in the present study we did not confirm our hypothesis 
that reduced vasomotor reactivity is associated with depressive disorders. The observed impairment of vasomotor reactivity in subjects with depressive symptoms was accounted for by cases with subthreshold depressive disorder. If this is not a chance finding, it suggests that cerebral microangiopathy may be less important in the more severely diseased and more often cause subthreshold depressive disorder. Interestingly, a recent neuropathological necropsy study also found no evidence of microvascular disease either locally or generally in the brain of depressed patients. ${ }^{41}$

\section{Limitations}

Some limitations of the study must be discussed. This was a cross sectional study and it cannot show whether the observed association with cerebrovascular changes precipitates or results from the depressive symptoms. Furthermore, we should consider whether selection influenced the outcome of the study. In the first place, transcranial Doppler measurements were not performed in all subjects participating in the third survey of the Rotterdam study; however, this omission was entirely random so it did not introduce bias. Second, transcranial Doppler measurements were unsuccessful in nearly one third of the subjects, and participants in whom transcranial Doppler measurements were unsuccessful were on average older and more often female. This was expected, as temporal bone acoustic thickness increases with age and in postmenopausal women, ${ }^{42}$ and this adversely affects the transmission of ultrasound. Hyperostosis, which is predominantly found in women, is also thought to preclude transcranial Doppler measurements. ${ }^{43}$ As old age and female sex are positively associated with depressive symptoms, persons with mood disorders were underrepresented in our study. This selection may have impeded the detection of modest associations. However, we think it unlikely that the observed relations between cerebral haemodynamic variables and depressive symptoms were a result of selection. There is no indication that haemodynamic changes differ in women or very old depressed subjects.

\section{Conclusions}

We have shown that depressive symptoms are associated with changes in both blood flow velocity and vasomotor reactivity. Our finding of reduced vasomotor reactivity suggests that vascular pathology may be a causal factor in subjects with subthreshold depressive disorder but not in DSM-IV depressive disorders. Furthermore, our data indicate that reduced cerebral blood flow velocity could be caused by reduced demand in depressed subjects and does not necessarily reflect microangiopathy.

\section{ACKNOWLEDGEMENTS}

The Rotterdam Study is supported in part by the NESTOR programme for geriatric research in the Netherlands (Ministry of Health and Ministry of Education), the Municipality of Rotterdam, the Netherlands Heart Foundation, and the Netherlands Organisation for Scientific Research (NWO). MMBB is a fellow of the Royal Netherlands Academy of Arts and Sciences.

\section{Authors' affiliations}

H Tiemeier, A Hofman, M M B Breteler, Department of Epidemiology \& Biostatistics, Erasmus Medical Centre Rotterdam, Rotterdam, Netherlands

S L M Bakker, P J Koudstaal, Department of Neurology, Erasmus Medical Centre Rotterdam

\section{REFERENCES}

1 Alexopoulos GS, Meyers BS, Young RC, et al. "Vascular depression" hypothesis. Arch Gen Psychiatry 1997;54:915-22.

2 Steffens DC, Helms M, Krishnan KR, et al. Cerebrovascular disease and depression symptoms in the cardiovascular health study. Stroke 1999;30:2159-66.
3 Baldwin RC, Tomenson B. Depression in later life. A comparison of symptoms and risk factors in early and late onset cases. Br J Psychiatry 1995; 167:649-52.

4 Alexopoulos GS, Meyers BS, Young RC, et al. Clinically defined vascular depression. Am J Psychiatry 1997;154:562-5.

5 Mendlewicz J. The age factor in depressive illness: some genetic considerations. J Gerontol 1976;31:300-3.

6 Mendlewicz J, Baron M. Morbidity risks in subtypes of unipolar depressive illness: differences between early and late onset forms. $\mathrm{Br} J$ Psychiatry 1981;139:463-6.

7 Krishnan KR, Hays JC, Blazer DG. MRI-defined vascular depression. Am J Psychiatry 1997; 154:497-501.

8 Ritchie K, Gilham C, Ledesert B, et al. Depressive illness, depressive symptomatology and regional cerebral blood flow in elderly people with sub-clinical cognitive impairment. Age Ageing 1999;28:385-91.

9 Lane RD, Reiman EM, Ahern GL, et al. Neuroanatomical correlates of happiness, sadness, and disgust. Am J Psychiatry 1997;154:926-33.

10 Schneider F, Gur RE, Mozley LH, et al. Mood effects on limbic blood flow correlate with emotional self-rating: a PET study with oxygen-15 labeled water. Psychiatry Res 1995;61:265-83.

11 Sackeim HA, Prohovnik I, Moeller JR, et al. Regional cerebral blood flow in mood disorders. I. Comparison of major depressives and normal controls at rest. Arch Gen Psychiatry 1990;47:60-70.

12 Silfverskiold $\mathbf{P}$, Risberg J. Regional cerebral blood flow in depression and mania. Arch Gen Psychiatry 1989;46:253-9.

13 Nobler MS, Pelton GH, Sackeim HA. Cerebral blood flow and metabolism in late-life depression and dementia. J Geriatr Psychiatry Neurol 1999;12:118-27.

14 Upadhyaya AK, Abou-Saleh MT, Wilson K, et al. A study of depression in old age using single-photon emission computerised tomography. $\mathrm{Br} J$ Psychiatry Suppl 1990;9:76-81.

15 Lesser IM, Mena I, Boone KB, et al. Reduction of cerebral blood flow in older depressed patients. Arch Gen Psychiatry 1994;51:677-86.

16 Aaslid R, Markwalder TM, Nornes H. Noninvasive transcranial Doppler ultrasound recording of flow velocity in basal cerebral arteries. J Neurosurg 1982;57:769-74.

17 Terborg C, Gora F, Weiller C, et al. Reduced vasomotor reactivity in cerebral microangiopathy: a study with near-infrared spectroscopy and transcranial Doppler sonography. Stroke 2000;31:924-9.

18 Newell DW, Aaslid R, eds. Transcranial Doppler. New York: Raven Press, 1992.

19 Sugimori H, Ibayashi S, Fujii K, et al. Can transcranial Doppler really detect reduced cerebral perfusion states? Stroke 1995;26:2053-60.

20 Franceschi $M$, Alberoni $M$, Bressi S, et al. Correlations between cognitive impairment, middle cerebral artery flow velocity and cortical glucose metabolism in the early phase of Alzheimer's disease. Dementia 1995; 6:32-8.

21 Braver $\mathbf{P}$, Kochs E, Werner $C$, et al. Correlation of transcranial Doppler sonography mean flow velocity with cerebral blood flow in patients with intracranial pathology. J Neurosurg Anesthesiol 1998;10:80-5.

22 Hofman A, Grobbee DE, de Jong PT, et al. Determinants of disease and disability in the elderly: the Rotterdam Elderly Study. Eur J Epidemiol 1991;7:403-22.

23 Heun R, Knappertz V, Kraemer G. Transcranial Doppler sonography in dementia of Alzheimer type. Dementia 1994;5:327-33.

24 Sawyer-Radloff L. The CES-D scale: a self-report depression scale for research in the general population. Appl Psychol Meas 1977;1:385-401.

25 Beekman AT, Deeg DJ, Van Limbeek J, et al. Criterion validity of the Center for Epidemiologic Studies Depression scale (CES-D): results from a community-based sample of older subjects in The Netherlands. Psychol Med 1997;27:231-5.

26 Blazer D, Burchett B, Service C, et al. The association of age and depression among the elderly: an epidemiologic exploration. J Gerontol 1991;46:M210-15

27 World Health Organisation Division of Mental Health. SCAN schedules for clinical assessment in neuropsychiatry, version 2.1. Distribution from training centers, 2nd ed. Geneva: World Health Organisation, 1997.

28 Fowkes FG, Housley E, Cawood EH, et al. Edinburgh Artery Study: prevalence of asymptomatic and symptomatic peripheral arterial disease in the general population. Int J Epidemiol 1991;20:384-92

29 Faravelli C, Marinoni M, Spiti R, et al. Abnormal brain hemodynamic responses during passive orthostatic challenge in panic disorder. Am J Psychiatry 1997; 154:378-83.

30 Ball S, Shekhar A. Basilar artery response to hyperventilation in panic disorder. Am J Psychiatry 1997; 154:1603-4.

31 Provinciali L, Minciotti P, Ceravolo G, et al. Transcranial Doppler sonography as a diagnostic tool in vascular dementia. Eur Neuro 1990;30:98-103.

32 Ries F, Horn R, Hillekamp J, et al. Differentiation of multi-infarct and Alzheimer dementia by intracranial hemodynamic parameters. Stroke 1993;24:228-35

33 Biedert S, Forstl H, Hewer W. Multiinfarct dementia vs Alzheimer's disease: sonographic criteria. Angiology 1995;46:129-35.

34 Hickie I, Scott E. Late-onset depressive disorders: a preventable variant of cerebrovascular disease? Psychol Med 1998:28:1007-13.

35 Berne RM, Winn HR, Rubio R. The local regulation of cerebral blood flow. Prog Cardiovasc Dis 1981;24:243-60.

36 Aaslid R. Cerebral hemodynamics. In: Newell DW, Aaslid R, eds. Trancranial Doppler. New York: Raven Press, 1992:49-55.

37 Droste DW, Harders AG, Rastogi E. A transcranial Doppler study of blood flow velocity in the middle cerebral arteries performed at rest and during mental activities. Stroke 1989;20:1005-11. 
38 Wood JH. Cerebral blood flow. Physiologic and clinical aspects. New York: McGraw-Hill, 1987

39 Ringelstein EB, Otis SM. Physiological testing of vasomotor reserve. In: Newell DW, Aaslid R, eds. Transcranial Doppler. New York: Raven Press, 1992:83-99.

40 Bakker SL, de Leeuw FE, de Groot JC, et al. Cerebral vasomotor reactivity and cerebral white matter lesions in the elderly. Neurology 1999;52:578-83.
41 Thomas AJ, Ferrier IN, Kalaria RN, et al. A neuropathological study of vascular factors in late-life depression. J Neurol Neurosurg Psychiatry $2001 ; 70: 83-7$

42 Bass A, Krupski WC, Schneider PA, et al. Intraoperative transcranial Doppler: limitations of the method. J Vasc Surg 1989;10:549-53.

43 Fujioka KA, Douville CM. Anatomy and freehand examination techniques. In: Newell DW, Aaslid R, eds. Transcranial Doppler. New York: Raven Press, 1992:9-32

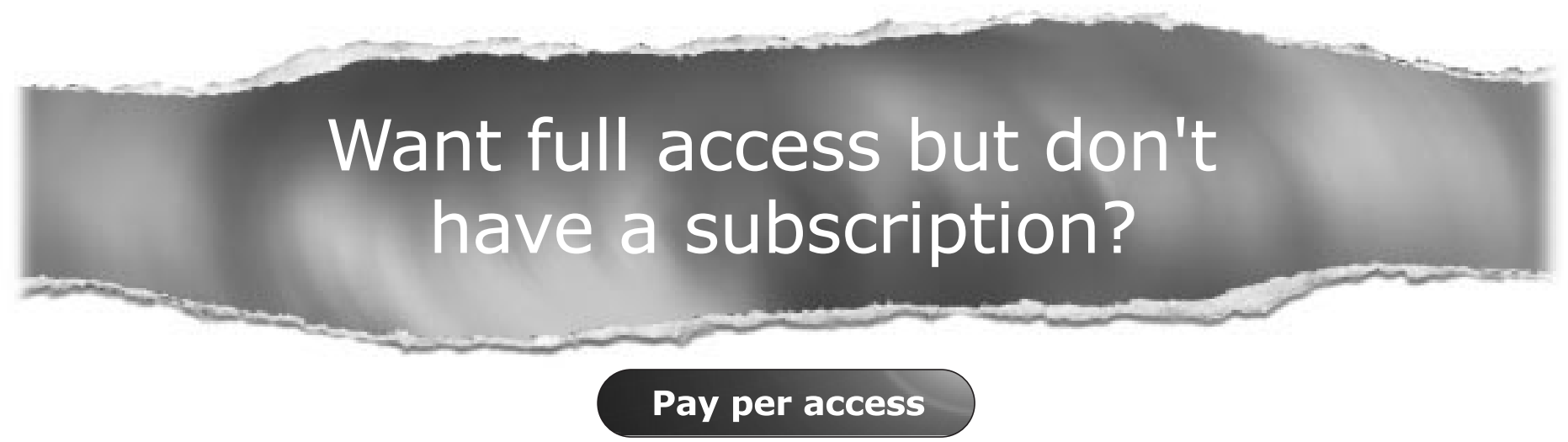

For just US\$25 you can have instant access to the whole website for 30 days. During this time you will be able to access the full text for all issues (including supplements) available. You will also be able to download and print any relevant pdf files for personal use, and take advantage of all the special features Journal of Neurology, Neurosurgery, and Psychiatry online has to offer.

www.jnnp.com 